HU-EP-04/62

\title{
Strong Coupling Dynamics of the Higgs Branch: Rolling a Higgs by Collapsing an Instanton
}

\author{
Zachary Guralnik* \\ Institut für Physik \\ Humboldt-Universität zu Berlin \\ Newtonstraße 15 \\ 12489 Berlin, Germany
}

\begin{abstract}
We construct the dual supergravity description of strongly coupled, large $N$, eightsupercharge gauge theories with fundamental hypermultiplets at points on the mixed CoulombHiggs branch. With certain assumptions about unknown couplings of D-branes to supergravity, this construction gives the correct metric on the hypermultiplet (Higgs-branch) component the moduli space, which decouples from the vector multiplet (Coulomb-branch) moduli. Going beyond the geodesic approximation, we find that the dynamics of a hypermultiplet VEV rolling towards a singularity on the Higgs component of the moduli space is sensitive to the vector multiplet moduli. The dual description of the approach to the singularity involves collapsing "instantons" of a non-Abelian Dirac-Born-Infeld theory in a curved background. In general, we find a decelerating approach to the singularity, although the manner of deceleration depends on the vector multiplet moduli. Upon introducing a potential on the Higgs branch of a four dimensional $\mathcal{N}=2$ theory coupled to gravity, this deceleration mechanism might lead to interesting inflating cosmologies analogous those studied recently by Alishahiha, Silverstein and Tong.
\end{abstract}

*zack@physik.hu-berlin.de 


\section{Introduction}

The holographic relation between string theory and Yang-Mills theories [1-3] known as AdS/CFT duality has proven a powerful tool for studying strongly coupled large $N$ YangMills theories. In its original form, this duality was only applicable to a certain class of gauge theories with matter in adjoint representations. More recently, there have been numerous studies of holographic dualities relating string theory and gauge theories with fundamental representations. This work has focused largely on electrically confining "QCDlike" theories. Instead of considering electrically confining theories, we will construct backgrounds dual to points on the mixed Coulomb-Higgs branch of eight-supercharge Yang-Mills theories with fundamental representations, in various dimensions. This work is a sequel to [4], in which the AdS dual of the Higgs branch of a four-dimensional $\mathcal{N}=2$ theory was constructed.

The backgrounds we will describe can be used to study the strong coupling, large $N$ dynamics on the Higgs component of the moduli space. Some aspects of this dynamics are known exactly already, since the two derivative effective action on the Higgs component of the moduli space is not renormalized [5]. Realizing this non-renormalization theorem in the dual description provides a test of AdS/CFT duality and constrains unknown terms in the D-brane effective action, including non-minimal couplings to bulk fields. In some situations however, the two derivative effective action can not be expected to give a correct description of the dynamics. One such situation is time dependent motion towards a singularity of the moduli space. We will study this motion using the holographic dual description. Similar dynamics has been studied in the context of the Coulomb branch of $\mathcal{N}=4$ super Yang-Mills theory [6], and gives rise to interesting inflating cosmologies upon introduction of a potential on the Coulomb-branch and coupling to gravity $[7,8]$ (see also [9] for related work).

In [4], the AdS description of the Higgs branch of a four-dimensional $\mathcal{N}=2 \operatorname{Sp}(\mathrm{N})$ gauge theory with four fundamental hypermultiplets and one anti-symmetric hypermultiplet was constructed. This theory is conformal at the origin of moduli space and is dual to string theory in the background $A d S^{5} \times S^{5} / Z^{2}$ with D7-branes wrapping the $Z^{2}$ fixed surface (an O7-plane) [10,11], which has the geometry $A d S^{5} \times S^{3}$. In [4], it was argued that the D7-brane equations of motion are solved by field strengths which are self dual with respect to a flat four dimensional metric, despite the curved background and the infinite number of higher dimension operators in the D7-brane action, which includes non-minimal couplings to bulk fields. These instanton solutions correspond to points on the Higgs branch, in keeping with the known one-to-one map between the ADHM constraints [12] used to construct instantons and the F and D-flatness conditions which, modulo gauge transformations, determine the Higgs branch [13,14] (see also [15] for a review). These instantons may be interpreted as D3-branes dissolved within the D7-brane [17]. Avoiding a back-reaction due to dissolved instantons requires that the instanton number, or number of dissolved D3-branes, is small compared to $N$. For this reason, the construction of [4] only applies to a portion of the Higgs branch.

We will generalize the construction of [4] to include parts of the mixed CoulombHiggs branch in eight supercharge theories in various dimensions. We will specifically consider eight supercharge theories arising from systems of $N$ Dp branes coincident with 
$N_{f} \mathrm{Dp}+4$ branes, with $p \leq 3$. For $N \rightarrow \infty$ with large 't Hooft coupling and $N_{f}$ fixed, these theories are dual to string theory in the near horizon geometry of the Dp-branes, with $N_{f}$ appropriately embedded probe $\mathrm{Dp}+4$ branes.

To two derivative order, the effective action on the Higgs branch is given exactly by the tree level result [5]. Furthermore the corresponding metric on the moduli space is equivalent to the metric which describes slowly varying "instantons" of super Yang-Mills theory in $p+5$ flat dimensions [13-15]. At strong coupling, AdS/CFT duality relates the effective action on the Higgs branch to the dynamics of slowing varying instantons in the Dp+4-brane theory, rather than flat space super Yang-Mills. Nevertheless, it was argued in [4] that (for $p=3$ ) the result is the same at two derivative order, provided certain constraints on unknown terms of the non-Abelian Dirac-Born-Infeld action in a curved background are satisfied. In fact, the non-renormalization of the metric on the Higgs branch implies that these constraints must be satisfied.

The non-renormalization of the metric on the Higgs branch follows from the local factorization the moduli space into a hypermultiplet and a vector-multiplet component, and the fact that the gauge coupling can be viewed as a component of a background vector multiplet [5]. We will see that the factorization of the moduli space into Coulomb and Higgs components is realized in the AdS description of the mixed Coulomb-Higgs branch.

The low energy dynamics of the Higgs branch has properties which may lead to interesting cosmologies upon introduction of a potential and coupling to gravity. These properties are similar to those discussed in $[7,8]$, where a mechanism for slow roll inflation in the presence of a steep potential was discovered in the context of a strongly coupled gauge large $\mathrm{N}$ gauge theory, specifically a deformation of $\mathcal{N}=4$ super Yang-Mills coupled to gravity. This mechanism arises from the higher derivative structure of the effective action on the Coulomb branch, which gives rise to an upper bound on velocity in the moduli space. The upper bound follows from causality in the AdS description [6], and implies that a certain time dependent Coulomb branch VEV rolling towards a singularity in the moduli space will decelerate. Upon suitably deforming the theory and coupling to gravity, there are cosmological solutions in which this time dependent modulus plays the role of the inflaton. A natural question is whether deceleration is a generic feature of time dependent moduli approaching a singularity in the moduli space.

Using the dual supergravity description, we will study the dynamics of time dependent Higgs branch moduli, keeping the Coulomb branch moduli fixed. In the geodesic, or "moduli space," approximation obtained by truncating to the two derivative effective action, this dynamics is insensitive to the Coulomb branch moduli and does not exhibit deceleration near singularities. However, the geodesic approximation is not always reliable, due to important corrections from integrating out states which become light as one approaches a singularity. We will find a deceleration mechanism for Higgs branch moduli approaching a singularity. The manner of deceleration depends on the Coulomb-branch moduli, and does not always follow from causality in the dual supergravity description.

We will focus on motion towards a singularity of the Higgs branch which is dual to a shrinking instanton. No attempt will me made to compute the full effective action including higher derivatives. Instead we will consider time dependent solutions of the $\mathrm{Dp}+4$ equations of motion which have instanton number one and possess the usual symmetries 
of the static one-instanton solution. Since the time dependent solutions are not self-dual, it is not obvious how to precisely identify the corresponding points on the Higgs branch. One way to do a more precise analysis would be to integrate out fluctuations transverse to the instanton moduli space (giving the effective action). Nevertheless, without exactly identifying points in the moduli space, qualitative properities of the evolution will be clear. The geodesic approximation, which is given by the two derivative effective action, incorrectly predicts that a shrinking instanton will collapse at a constant rate. Although the $\mathrm{Dp}+4$ equations of motion admit the usual static instanton solutions, and the two derivative effective action on the instanton moduli space is the same as that of flat space Yang-Mills theory, the equations describing a collapsing instanton differ from those of flat space Yang-Mills theory. Furthermore, these equations depend on parameters dual to Coulomb-branch moduli.

For vanishing Coulomb-branch moduli, there are decelerating solutions, much like those discussed in [6-8], for which deceleration follows from causality in the supergravity description. In this case it takes infinite time to reach the singularity at the origin of the Higgs branch. For certain non-vanishing Coulomb moduli there is also a deceleration mechanism. However, once the Higgs VEV becomes small compared to the Coulomb branch moduli, deceleration takes a different form which follows from particle production rather than a causal speed limit in the dual description. In this case deceleration is not strong enough to prevent reaching the small instanton singularity in a finite time. The rate of instanton collapse vanishes at the time of collapse and the kinetic energy of the collapse gets converted into the energy of modes transverse to the instanton moduli space, which are dual to states which become light at the origin of the Higgs branch. It is conceivable that, in a cosmological setting, this could be interpreted as reheating.

The organization of this article is as follows. In section 2, we review basic properties of the Higgs branch of eight supercharge theories arising from Dp-Dp+4 systems, In section 3, we discuss the supergravity description of a portion of the mixed CoulombHiggs branch. In section 4 we show that this description produces the correct metric on Higgs component of the moduli space. In section 5, we use the supergravity description to study time dependent solutions, going beyond the geodesic approximation. In section 6 , we conclude and discuss open problems.

\section{The Higgs branch of $\mathrm{Dp}-\mathrm{Dp}+4$ systems}

We wish to study eight-supercharge Yang-Mills theories in $\mathrm{p}+1$ dimensions corresponding to $\mathrm{Dp}-\mathrm{Dp}+4$ systems. For $p<3$ we will consider $N$ Dp-branes and $N_{f} \mathrm{Dp}+4$-branes, such that the $p+1$ dimensional theory has $U(N)$ gauge symmetry and contains, besides the vector-multiplet, one adjoint hypermultiplet and $N_{f}$ fundamental hypermultiplets. The fundamental hypermultiplets arise from strings stretched between the Dp-branes and $\mathrm{Dp}+4$-branes. Specifically, we take the $\mathrm{Dp}+4$-branes to extend in directions $x^{0 \cdots p+4}$ and the Dp-branes to extend in directions $x^{0 \cdots p}$. For $p=3$, the gauge theory arising from the D3-D7 configuration has a Landau pole, reflecting an un-cancelled tadpole in the string theory background. This pathology seems to avoided in the strict $N=\infty$ limit at finite $N_{f}$, in which the beta function for the 't Hooft coupling vanishes and the back-reacted geometry involves a D7-brane wrapping a contractible $S^{3}$ such that there 
is no net D7-charge [16]. Alternatively one can consider the case in which there are 4 D7-branes coincident with an O7-plane, such that one has a consistent tadpole free string background. This yields a conformal gauge theory even at finite $N$ (see [10,11]) with $\mathrm{Sp}(\mathrm{N})$ gauge symmetry and $\mathrm{SO}(8)$ flavour symmetry, one hypermultiplet in the antisymmetric representation and four hypermultiplets in the fundamental representation.

For definiteness, we will focus on the $\mathrm{U}(\mathrm{N})$ theories. The analysis can be generalized to other cases. The superpotential is

$$
W=\tilde{Q}_{i} X Q_{i}+\operatorname{tr} X[Y, Z]
$$

where $Q_{i}$ and $\tilde{Q}_{i}$ comprise fundamental hypermultiplets labeled by the flavor index $i, Y$ and $Z$ belong to an adjoint hypermultiplet, and $X$ belongs to the vector multiplet. There must be at least two flavors for a Higgs branch to exist. We will use lowercase letters to denote the bottom components of chiral superfields. On the Higgs branch, the vector multiplet moduli $x$ vanish while $q_{i}$ and $\tilde{q}_{i}$ have non-zero expectation values. There are also mixed Coulomb-Higgs vacua, for which $q_{i}, \tilde{q}_{i}$ and $x$ all have non-zero expectation values.

In the string theory realization of the theories which we consider, the $\mathrm{Dp}+4$-branes will always be taken to be coincident, while the Dp-branes (before taking a near horizon limit) may be at various locations in the transverse directions $x^{p+5} \cdots x^{9}$, which correspond to various Coulomb-branch moduli. Mixed Coulomb-Higgs vacua exist whenever some of the Dp-branes are coincident with the $\mathrm{Dp}+4$-branes. We will specifically consider the large $N$ limit in which in a fixed number $k$ of the Dp-branes are coincident with the $\mathrm{Dp}+4$-branes while the remaining $N-k$ Dp-branes are a distance $M \alpha^{\prime}$ from the $\mathrm{Dp}+4$-branes. This corresponds to vacua with

$$
x=\left(\begin{array}{cccccc}
M & & & & & \\
& \ddots & & & & \\
& & M & & & \\
& & & 0 & & \\
& & & & \ddots & \\
& & & & & 0
\end{array}\right) .
$$

for which the F-flatness equations $\tilde{q}_{i} x=x q_{i}=0$ permit fundamental hypermultiplet expectation values in which only the last $\mathrm{k}$ entries of $q$ and $\tilde{q}$ are non-zero;

$$
q_{i}=\left(\begin{array}{c}
0 \\
\vdots \\
0 \\
\alpha_{i 1} \\
\vdots \\
\alpha_{i k}
\end{array}\right) \quad \tilde{q}_{i}=\left(\begin{array}{llllll}
0 & \cdots & 0 & \beta_{i 1} & \cdots & \beta_{i k}
\end{array}\right)
$$

There are additional $\mathrm{F}$ and D-flatness constraints which we have not explicitly written.

Nonzero entries in (2.3) physically correspond to Dp-branes which are coincident with and dissolved within the $\mathrm{Dp}+4$-branes. Dissolved Dp-branes can be viewed as instantons 
in the $p+5$ dimensional world-volume theory on the $\mathrm{Dp}+4$ branes [17], due to the WessZumino coupling

$$
S_{W Z}=T_{p+4}\left(2 \pi \alpha^{\prime}\right)^{2} \int C^{(p+1)} \wedge \operatorname{tr} F \wedge F .
$$

In fact, there is a well known exact map between the moduli space of Yang-Mills instantons and the Higgs branch of the $p+1$ dimensional theory arising on the $\mathrm{Dp}-\mathrm{Dp}+4$ intersection. The ADHM constraints from which instantons are constructed [12] are equivalent to the $\mathrm{F}$ and $\mathrm{D}$ flatness equations [13-15] of the $p+1$ dimensional theory.

The metric on the Higgs branch is known to be tree level exact. [5]. A brief summary of the argument behind this non-renormalization is as follows. The Kahler potential is a function $K\left(h_{i}, \tilde{h}_{i}, \phi, h_{i}^{\dagger}, \tilde{h}_{i}^{\dagger}, \phi^{\dagger}\right)$ of the hypermultiplet moduli, collectively denoted by $h_{i}, \tilde{h}_{i}$, and the vector multiplet moduli $\phi$. Lorentz invariance together with $\mathcal{N}=2$ supersymmetry requires that $K=K_{H}\left(h_{i}, h_{i}^{\dagger}\right)+K_{V}\left(\phi, \phi^{\dagger}\right)$, such that the moduli space is locally a product of Higgs-branch and Coulomb-branch components. Finally, the gauge coupling can be viewed as a component of a background vector multiplet, on which $K_{H}$ does not depend.

For the theories associated with the $\mathrm{Dp}-\mathrm{Dp}+4$ system, the Hyperkähler metric on the Higgs branch is equivalent to that which descibes slowly varying "instantons" in eight dimensional super Yang-Mills theory [13-15]. These instantons correspond to field

strengths which are self-dual with respect to the flat metric in the directions $x^{4,5,6,7}$. The effective action for slowly varying instantons takes the form,

$$
\int d x^{0} \cdots d x^{3} G_{i j}(\mathcal{M}) \partial_{\mu} \mathcal{M}^{i} \partial^{\mu} \mathcal{M}^{j}
$$

where $\mathcal{M}^{i}$ are the instanton moduli, which are allowed to depend on the coordinates $x^{0,1,2,3}$, and $G_{i j}$ is the metric on the moduli space.

\section{Supergravity description of the Higgs branch}

For $N \rightarrow \infty$ with $N_{f}$ fixed and 't Hooft coupling $\lambda=g^{2} N>>1$, the super Yang-Mills theory associated with the $\mathrm{Dp}-\mathrm{Dp}+4$ system is dual to supergravity in the near horizon geometry of the $\mathrm{N}$ Dp-branes, with the $\mathrm{Dp}+4$ branes treated as probes. The near horizon geometry of the Dp-branes is

$$
\begin{aligned}
& d s^{2}=\alpha^{\prime}\left[H(r)^{-1 / 2} \eta_{\mu \nu} d x^{\mu} d x^{\nu}+H(r)^{1 / 2} \sum_{a=1}^{9-p}\left(d y^{a}\right)^{2}\right] \\
& C_{01 \cdots p}=\alpha^{\prime 2} H(r)^{-1} \\
& e^{-2\left(\phi-\phi_{\infty}\right)}=\alpha^{\prime 3-p} H(r)^{(p-3) / 2}, \quad e^{\phi_{\infty}}=g_{s},
\end{aligned}
$$

where $\mu=0 \cdots p, \eta_{\mu \nu}$ is the $p+1$ dimensional minkowski metric, and

$$
\begin{aligned}
r^{2} & =\sum_{a=1}^{9-p}\left(y^{a}\right)^{2}, \quad H(r)=\frac{d_{p} \lambda}{r^{7-p}}, \quad d_{p}=2^{7-2 p} \pi^{\frac{9-3 p}{2}} \Gamma\left(\frac{7-p}{2}\right), \\
\lambda & =(2 \pi)^{p-2} \alpha^{(p-3) / 2} g_{s} N .
\end{aligned}
$$


This supergravity background is reliable (see [18]) in the region

$$
\left(\lambda N^{\frac{4}{p-7}}\right)^{\frac{1}{3-p}}<<r<<\lambda^{\frac{1}{3-p}} .
$$

The $\mathrm{Dp}+4$ probes which gives rise to hypermultiplets with mass $M$ are localized in the geometry (3.1) at

$$
\begin{aligned}
& y^{a}=0 \text { for } 5 \leq a \leq 8-p, \\
& y^{9-p}=M .
\end{aligned}
$$

There must be at least two $\mathrm{Dp}+4$ branes, corresponding to a minimum of two flavors, for a Higgs branch to exist. The number of $\mathrm{Dp}+4$-branes must be held fixed in the large $N$ limit to avoid consideration of the back reaction. The induced metric on the $\mathrm{Dp}+4$-branes is

$$
d s^{2}=\alpha^{\prime}\left[H^{-1 / 2}(r) \eta_{\mu \nu} d x^{\mu} d x^{\nu}+H^{1 / 2}(r) d y^{m} d y^{m}\right]
$$

with $m=1 \cdots 4$ and $r^{2}=y^{m} y^{m}+M^{2}$. For $p=3$ this geometry is $A d S^{5} \times S^{3}$ if $M=0$, or asymptotically $A d S^{5} \times S^{3}$ when $M \neq 0$. The stability of such embeddings was discussed in [16], where it was shown that the Breitenlohner-Freedman bound is satisfied (saturated in fact), while the supersymmetry of the embedding was explicitly shown in [19]. For $p<3$, we expect that the embedding (3.4) remains supersymmetric although, to our knowledge, this has not been explicitly demonstrated.

The geometry associated with the mixed Coulomb-Higgs branch vacua (2.2), (2.3) is also (3.1) with the embedding (3.4), provided that the number, $k$, of vanishing diagonal entries in (2.2) is fixed in the large $N$ limit. This corresponds to a configuration of $k$ Dp-branes coincident with Dp+4-branes, in the near horizon geometry (3.1) arising from $N-k \mathrm{D}$-branes at a non-zero distance from the $\mathrm{Dp}+4$-branes. (3.4). At generic points in the Higgs-branch component of the moduli space, the $\mathrm{k} \mathrm{Dp}$-branes are dissolved in the $\mathrm{Dp}+4$-branes. The effective action of the $D p+4$ branes is a $p+5$-dimensional nonAbelian Dirac-Born-Infeld theory in a curved space. Nevertheless, the correspondence between instantons and the Higgs branch leads one to expect that conventional YangMills instantons should also solve the $\mathrm{Dp}+4$ equations of motion.

The $\mathrm{Dp}+4$ brane action takes the form

$$
\begin{aligned}
S & =T_{p+4} \int \sum_{r} C^{(r)} \wedge \operatorname{tr} e^{2 \pi \alpha^{\prime} F} \wedge\left(\frac{\hat{\mathcal{A}}\left(4 \pi^{2} \alpha^{\prime} R_{T}\right)}{\hat{\mathcal{A}}\left(4 \pi^{2} \alpha^{\prime} R_{N}\right)}\right)^{1 / 2} \\
& -T_{p+4} \int d^{p+5} \xi \sqrt{g} e^{-\left(\phi-\phi_{\infty}\right)}\left(2 \pi \alpha^{\prime}\right)^{2} \frac{1}{2} \operatorname{tr}\left(F_{A B} F^{A B}\right)+\cdots,
\end{aligned}
$$

where we have not written terms involving fermions and scalars. The action (3.6) is the sum of a Wess-Zumino term ${ }^{1}$, a Yang-Mills term, and an infinite number of corrections

\footnotetext{
${ }^{1}$ The "A-roof genus" $\hat{\mathcal{A}}$ appearing in the Wess-Zumino term (see [20]) has an expansion in terms of even powers of $R_{T}$ and $R_{N}$, which are pull-backs of the Reimann curvature two-form to the tangent and normal bundle respectively.
} 
at higher orders in $\alpha^{\prime}$, indicated by $\cdots$ in (3.6). Even in flat space, little is known about these corrections in the non-Abelian case, with the exception of a few of the leading order terms. Even less is known about higher dimension non-minimal couplings of Dbrane gauge fields to bulk fields such as the curvature, Ramond-Ramond field strengths, and derivatives of the dilaton.

Let us now consider turning on gauge fields $A_{m}\left(y^{n}\right)$, where the indices $m, n=1 \cdots 4$ are associated with the coordinates $y^{m}$ appearing in the induced metric (3.5) on the $\mathrm{Dp}+4$-brane. At leading order in the $\alpha^{\prime}$ expansion, field strengths $F_{m n}$ which are selfdual with respect to the flat four dimensional metric $d s^{2}=d y^{m} d y^{m}$ solve the equations of motion due to a conspiracy between the Wess-Zumino and Yang-Mills term. Inserting the explicit form of the induced metric, Ramond-Ramond potential, and dilaton into the leading terms of the $\mathrm{Dp}+4$ action (3.6) gives,

$$
\begin{aligned}
S & =\frac{N}{(2 \pi)^{6} \lambda} \int d^{p+1} x d^{4} y H(r)^{-1} \operatorname{tr}\left(F_{m n} F_{m n}-\frac{1}{2} \epsilon_{m n r s} F_{m n} F_{r s}\right) \\
& =\frac{N}{2(2 \pi)^{6} \lambda} \int d^{p+1} x d^{4} y H(r)^{-1} \operatorname{tr} F_{-}{ }^{2},
\end{aligned}
$$

where $r^{2}=\sum_{m=1}^{4}\left(y^{m}\right)^{2}+M^{2}, F_{-}=\frac{1}{2}\left(F_{m n}-\frac{1}{2} \epsilon_{m n r s} F_{r s}\right)$. We have written the Dp+4brane tension explicitly in terms of $\alpha^{\prime}$ and parameters of the dual $p+1$ dimensional gauge theory:

$$
T_{p+4}=\frac{1}{g_{s}(2 \pi)^{p+4} \alpha^{\prime(p+5) / 2}}=\frac{N}{(2 \pi)^{6} \alpha^{\prime 4} \lambda}
$$

where $\lambda=g_{Y M}^{2} N$ is the 't Hooft coupling. Field strengths for which $F_{-}=0$ are self-dual with respect to the flat metric $d y^{m} d y^{m}$ and manifestly solve the equations of motion.

The self dual field strengths correspond to points on the Higgs component of the moduli pace, while the parameter $M$ is a Coulomb-branch modulus. Note that the same self-dual solutions exist even if one does not take the near horizon limit, such that $H(r)=\alpha^{\prime 2}+d_{p} \lambda / r^{7-p}$. The usual flat space instantons continue smoothly into the near horizon region. Thus we expect that the the map between instanton moduli in the supergravity description and points on the Higgs component of the moduli space is the usual one. Strictly speaking, we can only describe a portion of the Higgs branch in this way, since the number of dissolved D3-branes is held fixed in the large $N$ limit to avoid having to consider back reaction.

The exact correspondence between the Higgs branch and Yang-Mills instantons leads one to expect that self-dual field strengths should remain solutions of the $\mathrm{Dp}+4$-brane action to all order in the $\alpha^{\prime}$ expansion. This implies constraints on various unknown terms of the non-abelian Dirac Born Infeld action in a curved background (see [4]). These constraints are somewhat beside the point of this paper, but are worth mentioning briefly. Among the constraints is one which applies to terms quadratic in the world-volume field strengths, of the general form $f\left(R, \mathcal{F}^{(p+2)}, \phi\right) F^{2}$. Here $f\left(R, \mathcal{F}^{(p+2)}, \phi\right)$ depends on the pull-backs of the bulk fields which are nonvanishing in the background (3.1), as well as their derivatives. We have not written any explicit contractions of Lorentz indices, of which there are numerous possibilities. At order $\alpha^{\prime}$, terms of the form $R^{2} F^{2}$ have been 
discussed in $[21,22]$. Assuming $F_{-}=0$ connections solve the equations of motion, there are instanton solutions for which the field strength is locally arbitrarily small compared to the bulk fields, and one can expand in the field strength. At quadratic order in field strengths the CP odd Wess-Zumino term proportional to $\int H(r)^{-1} F^{*} F$ is exact and receives no corrections at any order in $\alpha^{\prime}$. In order to preserve the $F^{-}=0$ solutions, the quadratic CP even term must be of the form $\int H(r)^{-1} F^{2}$ with exactly the same coefficient. As discussed above, this is already the case at leading order in $\alpha^{\prime}$. Thus at every higher order in $\alpha^{\prime}$, the terms of the form $\alpha^{\prime n} f_{n}\left(R, \mathcal{F}^{(p+2)}, \phi\right) F^{2}$ must sum to zero when the bulk fields are set to the background values (3.1). These constraints are very similar to constraints on the non-Abelian Dirac-Born-Infeld action in flat space which have been found by requiring that stable-holomorphic bundles solve the equations of motion [23].

Assuming that terms of the form $f\left(R, \mathcal{F}^{(p+2)}, \phi\right) F^{2}$ collectively sum to zero, the action to order $\alpha^{\prime 2}$ is given by

$$
\begin{aligned}
S= & T_{p+4} \int C^{(p+1)} \wedge F \wedge F \\
& +T_{p+4} \int \sqrt{-g} e^{-\left(\phi-\phi_{\infty}\right)} \operatorname{tr}\left[F^{A B} F_{A B}+\alpha^{\prime 2}\left(\frac{1}{24} F_{A B} F^{B C} F_{C D} F^{D A}\right.\right. \\
& \left.\left.+\frac{1}{12} F_{A B} F^{B C} F^{D A} F_{C D}-\frac{1}{48} F_{A B} F^{B A} F_{C D} F^{D C}-\frac{1}{96} F_{A B} F_{C D} F^{B A} F^{D C}\right)\right],
\end{aligned}
$$

where the $F^{4}$ terms were computed in [24-27]. For the embedding (3.4) in the background (3.1), with non-zero field strengths in the $y^{m}$ directions only, this action becomes

$$
\begin{aligned}
S=\frac{N}{2(2 \pi)^{6} \lambda} \int d^{p} x d^{4} y \operatorname{tr} & {\left[H^{-1}(r) F_{m n}^{-} F_{m n}^{-}\right.} \\
- & \left.\frac{1}{12} H^{-2}(r)\left(2 F_{m n}^{+} F_{m n}^{+} F_{r s}^{-} F_{r s}^{-}+F_{m n}^{+} F_{r s}^{-} F_{m n}^{+} F_{r s}^{-}\right)\right],
\end{aligned}
$$

where $r^{2}=y^{m} y^{m}+M^{2}$, and we have written $F_{m n}$ in terms of self-dual and anti-selfdual components, $F_{m n}^{ \pm} \equiv \frac{1}{2}\left(F_{m n} \pm \frac{1}{2} \varepsilon_{m n r s} F_{r s}\right)$. Since all terms in (3.10) involve two factors of $F^{-}$, field strengths for which $F^{-}=0$ are manifestly solutions. In light of the correspondence between instantons and the Higgs branch, this should hold for the full Dirac-Born-Infeld action.

\section{The metric on the Higgs Branch}

To two derivative order, the effective action on the Higgs branch of the eight supercharge $p+1$ dimensional theory is tree level exact, and is known to be equivalent to the action describing the geodesic approximation [28] for slowly varying "instantons" in a $p+5$ dimensional Yang Mills theory. These instantons are localized in the directions $y^{m} \sim$ $x^{p+1} \cdots x^{p+4}$ and, in the static case, have no dependence on the coordinates $x^{\mu} \sim x^{0} \cdots x^{p}$. The associated gauge connections have the form $A_{m}=A_{m}^{\text {inst }}\left(y^{n}, \mathcal{M}_{i}\right)$, where $\mathcal{M}_{i}$ are the instanton moduli. There are slowly varying approximate solutions in which the moduli 
$\mathcal{M}_{i}$ depend on the coordinates $x^{\mu}$. The effective action governing these moduli has the form

$$
S=\int d^{p+1} x G_{i j}(\mathcal{M}) \partial_{\mu} \mathcal{M}^{i} \partial^{\mu} \mathcal{M}^{j}
$$

and is equivalent to the two-derivative effective action on Higgs the branch, where the $\mathcal{M}_{i}$ are interpreted as scalars parameterizing the Higgs-branch.

In the dual description of the $p+1$ dimensional theory given by the geometry (3.1) with Dp+4-branes embedded according to (3.4), one can compute the metric on the Higgs branch by finding the action for slowly varying instantons on the Dp+4-branes. In spite of the curved background and infinite number of higher dimension operators in the $\mathrm{Dp}+4$-brane action, the result must be identical to that obtained from conventional Yang-Mills theory in $p+5$ flat dimensions.

The metric can be extracted from the equations which configurations of the form

$$
A_{m}=A_{m}^{\text {inst }}\left(y^{n}, \mathcal{M}_{i}\left(x^{\alpha}\right)\right), \quad A_{\mu}=\Omega_{i} \partial_{\mu} \mathcal{M}_{i}\left(x^{\alpha}\right)
$$

must satisfy in order to solve the equations of motion at leading order in a $\partial_{\mu}$ expansion (see [15] for a review). For this purpose, the relevant terms in the Dirac Born Infeld action are those involving two Greek indices. To order $\alpha^{\prime 2}$, which becomes $\mathcal{O}(1 / \lambda)$ in the background we consider, the terms of this type arising from the action (3.9) are,

$$
\begin{aligned}
S= & \frac{N}{(2 \pi)^{6} \lambda} \int d^{p+5} \xi \frac{1}{4} \operatorname{tr}\left(F_{\mu m} F_{\mu m}\right) \\
& +\frac{N}{(2 \pi)^{6} \lambda} \int d^{p+5} \xi H(r)^{-1} \frac{1}{12} \operatorname{tr}\left[F_{s \mu} F_{\mu n}\left(\left\{F_{n r}, F_{r s}\right\}-\frac{1}{2} \delta_{s n} F_{t u} F_{u t}\right)+\right. \\
& \left.\frac{1}{2}\left(F_{\mu n} F_{n r} F_{s \mu} F_{r s}+F_{\mu n} F_{r s} F_{s \mu} F_{n r}-\frac{1}{2} F_{\mu n} F_{r s} F_{n \mu} F_{s r}\right)\right]+\cdots
\end{aligned}
$$

Note that the term quadratic in $F$ is just that of Yang-Mills theory in $p+5$ flat dimensions; factors of the harmonic function $H(r)$ appearing in the metric (3.1) cancel in this term. This is the leading term in a large $\lambda$ expansion. Since $H^{-1} \sim r^{7-p} / \lambda$, the $F^{4}$ terms in (4.3) are subleading. However, the leading term should be the only term contributing to the metric on the Higgs branch, since flat space Yang-Mills gives the exact metric.

To see that the subleading term does not contribute, it is useful to rewrite it in terms of the self-dual and anti-self-dual parts of $F_{m n}$, giving

$$
\begin{aligned}
S= & \frac{N}{(2 \pi)^{6} \lambda} \int d^{p+5} \xi \frac{1}{4} \operatorname{tr}\left(F_{\mu m} F_{\mu m}\right) \\
+ & \frac{N}{(2 \pi)^{6} \lambda} \int d^{p+5} \xi H(r)^{-1} \frac{1}{48} \operatorname{tr}\left[F_{s \mu} F_{\mu n}\left(\left\{F_{n r}^{+}, F_{r s}^{-}\right\}+\left\{F_{n r}^{-}, F_{r s}^{+}\right\}\right)+\right. \\
& \left.\frac{1}{2}\left(F_{\mu n} F_{n r}^{+} F_{s \mu} F_{r s}^{-}+F_{\mu n} F_{n r}^{-} F_{s \mu} F_{r s}^{+}+F_{\mu n} F_{r s}^{+} F_{s \mu} F_{n r}^{-}+F_{\mu n} F_{r s}^{-} F_{s \mu} F_{n r}^{+}\right)\right]
\end{aligned}
$$

Hence, the subleading terms vanish when $F_{-}=0$. The calculation of the metric goes through as it does in flat space Yang-Mills. For the configuration (4.2), the equations of 
motion $\frac{\delta}{\delta A_{\mu}} S=0$ become

$$
\partial_{\mu} \mathcal{M}_{i}\left(D_{m} \frac{\delta A_{m}}{\delta \mathcal{M}_{i}}-D_{m} D_{m} \Omega_{i}\right)=0,
$$

which has a solution for $\Omega_{i}$ as a function of $y^{m}$ and $\mathcal{M}_{i}$. Taking this $\Omega_{i}$ and inserting (4.2) into the action (4.4) gives the metric on the Higgs branch;

$$
\partial_{\mu} \mathcal{M}^{i} \partial_{\mu} \mathcal{M}^{j} G_{i j}(\mathcal{M})=\frac{N}{(2 \pi)^{6} \lambda} \int d^{4} y \frac{1}{4} \operatorname{tr}\left(F_{\mu m} F_{\mu m}\right) .
$$

Note that the metric is insensitive to the embedding parameter $M$ which enters the action through the harmonic function $H(r)$ with $r^{2}=y^{m} y^{m}+M^{2}$. This parameter corresponds to an expectation value for a vector multiplet scalar. Insensitivity to $M$ reflects the fact that the moduli space factorizes locally into Higgs and Coulomb branch components [5].

In fact, all higher order contributions in the strong coupling expansion of the metric must vanish, since the flat space Yang-Mills result for the metric is exact. Note that we have obtained the correct metric at next to leading order without considering possible non-minimal couplings of D-brane fields to bulk fields. The absence of a contribution to the metric from these couplings yields another constraint on unknown bulk to brane couplings.

\section{Time dependent solutions: beyond the geodesic approximation}

We have argued that the AdS description of mixed Coulomb-Higgs vacua gives the correct two-derivative effective action on the Higgs component, corresponding to the geodesic motion of instantons in a flat space Yang-Mills theory. This result is insensitive to the expectation values of vector multiplet scalars, reflecting the local factorization of moduli space into Higgs and Coulomb parts. Nevertheless, we will see that the dynamics of a time dependent Higgs field rolling towards a singular point the moduli space is not necessarily described by the geodesic approximation, nor is it independent of the Coulomb branch expectation values.

At strong coupling, one particular approach to a singularity of the Higgs branch can be studied by considering the equations of motion of a collapsing instanton on the Dp +4 branes embedded in the geometry (3.1) according to (3.4). In principle, the AdS description allows one to compute the low energy effective action on the Higgs branch at strong 't Hooft coupling, including higher derivative terms, by integrating out modes transverse to the instanton moduli space. We leave this computation for the future. Instead, we will look for qualitative properties of approach to the singularity by examining solutions of the $\mathrm{Dp}+4$-brane equations of motion, without integrating out modes transverse to the instanton moduli space. Note that it does not make sense to integrate out these modes if there is production of particles (W-bosons and their superpartners) which become light as a Higgs scalar approach the singularity, in which case the effective action on the Higgs branch is not a good description. We will see that this circumstance arises when the Coulomb branch modulus $M$ is non-zero. 
Exact collapsing instanton solutions are not self-dual and are therefore not described by the ADHM construction. Without integrating out modes transverse to the instanton moduli space, it is not obvious how to map points on the Higgs branch, unless one works in an approximation in which the deviation from self-duality is small. For nonzero values of the Coulomb branch modulus $M$ and sufficiently small instanton size, we will be able to borrow results obtained in [29], in which such an approximation was used to describe collapsing instantons of Yang-Mills theory in five-dimensional Minkowski space-time. For vanishing Coulomb-branch moduli, we will find approximate collapsing instanton solutions which are rather far from self-duality. In this case we will only be able to make qualitative statements about the behavior of the Higgs expectation value.

\subsection{Causality in AdS}

As discussed in [6-8], the dynamics of Coulomb-branch moduli in large N, strongly coupled, $\mathcal{N}=4$ super Yang-Mills theory is constrained by causality in the dual AdS description. In particular there is an upper bound on the velocity with which moduli can roll towards a singularity, which decreases with proximity to the singularity. One might expect similar constraints to hold for the Higgs branch (or mixed Coulomb-Higgs branch) of the eight supercharge theories we have been considering. In this setting, the relevant part of the induced metric on the $\mathrm{Dp}+4$-branes is

$$
d s^{2}=\alpha^{\prime}\left[-H(r)^{-1 / 2} d t^{2}+H(r)^{1 / 2} \sum_{m=1}^{4} d y^{m} d y^{m}\right]
$$

with $r^{2}=y^{m} y^{m}+M^{2}$. For $M=0$, the point $y^{m}=0$ corresponds to the horizon $r=0$. Causality implies that it takes a pointlike object infinite coordinate time $t$ (corresponding to time in the dual $p+1$ dimensional gauge theory) to reach $y^{m}=0$. For $M \neq 0$, the point $y^{m}=0$ is not at the horizon and causality does not prevent pointlike objects from reaching this point in finite time. Although an instanton is not a pointlike object, it seems a natural supposition that the size of an instanton centered at the origin is also subject to causality constraints. For $M=0$, one would expect that it takes infinite time $t$ for an instanton to collapse, since the zero size instanton is localized at the horizon. We will see this expectation born out by some approximate collapsing instanton solutions. This behavior is very different from what is predicted by the geodesic approximation (two-derivative effective action) which gives collapse at a constant rate: $\ddot{\rho}=0$ where $\rho$ is the instanton size.

For $M \neq 0$, causality can not rule out collapse in finite time. Nevertheless, we will still find a deceleration mechanism which violates the geodesic approximation. This deceleration is unrelated to causality in the dual supergravity description, and is not strong enough to prevent collapse in a finite time. However the rate of collapse does vanish in the zero size limit. 


\subsection{Collapsing instantons}

The action for the $\mathrm{Dp}+4$ brane with the embedding (3.4) is

$$
\begin{aligned}
S= & \frac{N}{(2 \pi)^{6} \lambda} \int d^{p+5} \xi \operatorname{tr}\left(\frac{1}{2} H(r)^{-1} F_{m n}^{-} F_{m n}^{-}+2 F_{m \mu} F_{m \mu}+H(r) F_{\mu \nu} F_{\alpha \beta} \eta^{\mu \alpha} \eta^{\nu \beta}\right) \\
& +\cdots,
\end{aligned}
$$

where Greek indices indicate the coordinates $x^{0 \cdots p}$ and Roman indices indicate the coordinates $y^{1 \cdots 4}$. The higher dimension terms indicated by $\cdots$ involve arbitrarily high powers of $H(r)$ or $H(r)^{-1}$, depending on the numbers of Greek or Roman indices. Since $H(r)=\lambda / r^{7-p}$, a strong coupling expansion becomes manifest only after the rescaling $x^{\mu} \rightarrow \sqrt{\lambda} x^{\mu}, A_{\mu} \rightarrow A_{\mu} / \lambda$. After this rescaling, the supergravity metric has an overall $\sqrt{\lambda}$ in front, and (5.2) becomes

$$
\begin{aligned}
S & =\frac{N}{(2 \pi)^{6} \lambda^{2}} \int d^{p+5} \xi \operatorname{tr}\left(\frac{r^{7-p}}{2 d_{p}} F_{m n}^{-} F_{m n}^{-}+2 F_{m \mu} F_{m \nu} \eta^{\mu \nu}+\frac{d_{p}}{r^{7-p}} F_{\mu \nu} F_{\alpha \beta} \eta^{\mu \alpha} \eta^{\nu \beta}\right) \\
& +\mathcal{O}\left(\frac{1}{\lambda^{3}}\right),
\end{aligned}
$$

where $r^{2}=y^{m} y^{m}+M^{2}$. We will only consider the leading term in the strong coupling expansion.

Since we focus on the quadratic part of the action, the reader might incorrectly suspect that what we are doing is very different from that of [6-8], where behavior outside the geodesic approximation followed from considering the full Dirac-Born-Infeld action. In strongly coupled large $N \mathcal{N}=4$ super Yang-Mills theory, the dynamics of a particular Coulomb-branch modulus rolling towards a singularity of the moduli space is described by the effective action of a probe D3-brane in AdS,

$$
S=\int d^{4} \xi X^{4}\left(\sqrt{1-\frac{\lambda \dot{X}^{2}}{X^{4}}}-1\right)
$$

with the singularity lying at $X=0$. The geodesic approximation is $S \sim \int \dot{X}^{2}$, which is blind to the causal constraint $\sqrt{\lambda} \dot{X} \leq X^{2}$ arising from an infinite number of higher derivative corrections. It must be remembered that in the case of the Higgs branch, we have not yet integrated out modes which are transverse to the moduli space, after which one would also obtain an action with an infinite number of higher derivatives. Furthermore the action (5.4) also gives a leading contribution in a strong coupling expansion (seen more clearly after rescaling $x^{\mu} \rightarrow \sqrt{\lambda} x^{\mu}$ ).

To focus on time dependent solutions of (5.3) with no dependence on $x^{1 \cdots 3}$, it is sufficient to consider the five dimensional action

$$
S=\int d t d^{4} y \operatorname{tr}\left[F_{0 m} F_{0 m}-\frac{1}{2 d_{p}}\left(y^{m} y^{m}+M^{2}\right)^{(7-p) / 2} F_{m n}^{-} F_{m n}^{-}\right]
$$

This action has the usual static instanton solutions. The $\mathrm{SU}(2)$ single instanton solutions are

$$
A_{m}^{a}(y)=\frac{2 \eta_{m n}^{a}\left(y^{n}-Y^{n}\right)}{(y-Y)^{2}+\rho^{2}}
$$


where $m=1,2,3,4, a$ is a Lie algebra index, and $\eta_{n m}^{a}$ is the 't Hooft tensor;

$$
\eta_{m n}^{a}=-\eta_{n m}^{a}, \eta_{i j}^{a}=\epsilon_{a i j}, \eta_{i 4}^{a}=\delta_{i a} \text { with } i=1,2,3
$$

We will study time-dependant solutions with which preserve the symmetries of the single $S U(2)$ instanton centered at the origin and have instanton number one. Unlike the flat space Yang-Mills theory, the origin is a special point due to the curved geometry of the $\mathrm{Dp}+4$-brane embedding. Note that this fact is not visible in the metric on the instanton moduli space. We make the time dependant ansatz

$$
\begin{aligned}
A_{m}^{a} & =\eta_{n l}^{a} x^{l} \frac{f(v, t)}{v^{2}} \\
A_{0}^{a} & =0
\end{aligned}
$$

where $v^{2} \equiv y^{m} y^{m}$. The Gauss law constraint, $\frac{\delta}{\delta A_{0}} S=0$ is satisfied by this ansatz. The remaining equations of motion give ${ }^{2}$;

$$
\begin{gathered}
\left(-\partial_{t}^{2}+r^{7-p} \partial_{v}^{2}+\left(r^{7-p}+(7-p) v^{2} r^{5-p}\right) \frac{1}{v} \partial_{v}\right) f \\
+\frac{r^{7-p}}{v^{2}}\left(-4 f+6 f^{2}-2 f^{3}\right)+r^{5-p}\left(f^{2}-2 f\right)=0
\end{gathered}
$$

with $r^{2}=v^{2}+M^{2}$. We have rescaled coordinates to remove numerical factors of $d_{p}$.

\subsection{Rolling Higgs at the origin of the Coulomb branch}

Let us now examine solutions of (5.9) in the special case $M^{2}=0$ corresponding to the origin of the Coulomb branch. For $M^{2}=0$, (5.9) becomes

$$
\begin{array}{r}
\left(-\partial_{t}^{2}+v^{7-p} \partial_{v}^{2}+(8-p) v^{7-p} \frac{1}{v} \partial_{v}\right) f+v^{5-p} Q(f)=0 \\
\text { with } Q(f) \equiv-18 f+13 f^{2}-2 f^{3}+p\left(2 f-f^{2}\right)
\end{array}
$$

The usual static instanton solution is,

$$
f=\frac{2 r^{2}}{r^{2}+\rho^{2}},
$$

which interpolates between the zeros $f=0$ and $f=2$ of $Q(f)$. As long as a configuration $f(r, t)$ interpolates between these points at any fixed value of $t$, the instanton number $\frac{1}{16 \pi^{2}} \int F \wedge F$ is equal to one. It is not difficult to see that exact time dependent solutions can not be self-dual. Thus there is no exact solution of the form $f=2 r^{2} /\left(r^{2}+\rho(t)^{2}\right)$, although this may be taken as the leading term in an expansion valid for sufficiently slowly varying instanton size. While this form is suitable for determining the two derivative effective action, studying the corrections to the geodesic approximation requires considering configurations which are not self-dual.

\footnotetext{
${ }^{2}$ Some useful identities involving the 't Hooft tensor are written in the appendix.
} 
The map between the time dependent instanton solution and points on the Higgs branch is less clear than in the static case because the solutions are not described by the ADHM construction. One way to define the instanton size $\rho(t)$ is by the moment ${ }^{3}$ $\rho^{2} \sim \frac{1}{16 \pi^{2}} \int r^{2} F \wedge F$. Alternatively one can define $\rho(t)$ as the value of $r$ for which $f(r, t)=1$. We do not expect either of these definitions to map to points on the Higgs branch in the same way as the instanton size in the static case. However we will only be interested in qualitative behavior for which either definition can be taken as a crude measure of the dual Higgs VEV. Yet another possibility, which is more natural from the point of view of the usual AdS/CFT dictionary, is to define $\rho$ by the large $r$ asymptotics. In the static case, the leading large $r$ behavior of the field strength is $F_{m n}^{a} \sim 4 \rho^{2} \eta_{m n}^{a} / r^{4}$. However this definition will not prove useful for the approximate solution below, which is only valid at small $r$.

It is convenient to define $z \equiv \frac{2}{p-5} r^{(p-5) / 2}$ such that (5.10) becomes

$$
\left(-\partial_{t}^{2}+\partial_{z}^{2}+\frac{9-p}{p-5} \frac{1}{z} \partial_{z}\right) f+\frac{1}{z^{2}} \frac{4}{(p-5)^{2}} Q(f)=0
$$

Equation (5.12) has approximate collapsing instanton solutions;

$$
f(z, t) \approx \tilde{f}(z-t)
$$

where $\tilde{f}(z)$ interpolates between $f=0$ and $f=2$ in a region of sufficiently large $z$ (small r) such that $Q(\tilde{f}) / z^{2}<<\partial_{z}^{2} \tilde{f}$ and $\partial_{z} \tilde{f} / z<<\partial_{z}^{2} \tilde{f}$. This approximation is a good description of the instanton collapse as $t$ increases, but is not valid for sufficiently early times (negative $t$ ), since the conditions $Q(f) / z^{2}<<\partial_{z}^{2} f$ and $\partial_{z} f / z<<\partial_{z}^{2} f$ are violated. In terms of the coordinate $z$, the point at which $f=1$ propagates with velocity $\dot{z}=1$. Thus it takes infinite time $t$ for the instanton to collapse, which occurs when $\left.z\right|_{f=1}=\infty$. This solution corresponds to a spherical shell of topological charge moving at the speed of light. Restricting to fixed points $y^{m} /|y|$ on the sphere, the metric (5.1) is (for $M=0$ ) conformally equivalent to $d t^{2}-d z^{2}$.

The motion $\dot{z}=1$ of the collapsing instanton suggests a rolling Higgs

$$
\langle\tilde{q} q\rangle \approx r^{2} \text { with } \dot{r}=r^{(7-p) / 2} .
$$

Just as in [6-8], causality in the supergravity dual implies a deceleration mechanism. The VEV takes infinite time to roll to the singularity at the origin of the Higgs branch, provided that the Coulomb branch modulus $M$ vanishes. We emphasize that we can not be completely confident in the relation (5.14), because our solution is rather far from self dual. One way to verify this sort of behavior would be to computate the higher derivative terms in the effective action on the moduli space by integrating out the non self-dual modes in the Dirac-Born-Infeld action. This would give a more precise result, since one could accurately identify dual points on the Higgs branch.

By introducing a potential and coupling to (four dimensional) gravity, it may be possible to obtain inflating cosmologies similar to those discussed in $[7,8]$, except that the inflaton is a Higgs branch scalar rather than Coulomb branch scalar. The slow roll conditions would then be satisfied by virtue of of the same sort of deceleration mechanism, which follows from causality in the dual description.

\footnotetext{
${ }^{3}$ This is equal to the hyperkähler potential which, for a single static instanton localized at the origin, is proportional to the square of the instanton size
} 


\subsection{Rolling Higgs fields for non-zero Coulomb branch VEVs}

Let us now consider the case in which the Coulomb-branch modulus $M$ is non-vanishing. Despite the insensitivity of the Higgs branch metric to vector multiplet moduli, motion towards a singularity in the Higgs component of the moduli space depends on $M$.

For instanton sizes large compared to $M$, the time evolution should be approximately the same as for $M=0$. However, for sufficiently small instantons, with support in the region $y^{m} y^{m}<<M^{2}$, the relevant part of the $\mathrm{Dp}+4$ geometry is almost flat and the action (5.5) becomes;

$$
S \approx \int d t d^{4} y \operatorname{tr}\left[F_{0 m} F_{0 m}-\frac{1}{2 d_{p}} M^{7-p} F_{m n}^{-} F_{m n}^{-}\right]
$$

After a rescaling of coordinates to remove the factor $M^{7-p} / d_{p}$ the equations of motion are the same as those of Yang-Mills theory in flat five-dimensional space ${ }^{4}$. There is no longer a causal argument to prohibit the instanton from collapsing in a finite time; a zero size instanton is far from the horizon, since $y^{m} y^{m}=0$ corresponds to $r^{2}=M^{2}$.

After rescaling to remove the factor of $M^{7-p} / d_{p}$ in (5.15), the equations of motion for the ansatz (5.8), are

$$
\left(-\partial_{t}^{2}+\partial_{v}^{2}+\frac{1}{v} \partial_{v}\right) f+\frac{1}{v^{2}}\left(-4 f+6 f^{2}-2 f^{3}\right)=0 .
$$

These equations have been studied in [29] using a perturbative expansion appropriate for solutions sufficiently close to the static instanton solution;

$$
f(r, t)=\frac{2 r^{2}}{r^{2}+\rho(t)^{2}}+\epsilon(r, t) .
$$

where $\epsilon$ is orthogonal (with respect to a certain inner product) to the zero mode $\partial_{\rho}\left(\frac{2 r^{2}}{r^{2}+\rho(t)^{2}}\right)$ and has an expansion in time derivatives of $\rho$. For collapsing initial conditions, the authors of [29] found that the instanton collapses in finite time:

$$
\rho(t)=\frac{2}{3} \frac{t^{*}-t}{\sqrt{-\ln \left(t^{*}-t\right)}}
$$

where $t^{*}$ is the time of collapse.

Although the instanton collapses in finite time, the rate of collapse $\dot{\rho}$ vanishes at $t=t^{*}$. Energy conservation then requires that the kinetic energy associated with the time dependence of the instanton size must be converted into energy of modes transverse to the moduli space, i.e. modes contained in $\epsilon(r, t)$. In the dual gauge theory, the kinetic energy of the rolling Higgs field is converted entirely into the energy of particles which become light and are radiated during the approach to the Higgs branch singularity. Assuming that inflating cosmologies like those of $[7,8]$ can be realized during the part of collapse for which the Higgs VEV (instanton size) is large compared to $M$, then the part of the collapse for which the Higgs VEV is small compared to $M$ seems a natural candidate for reheating. We leave the problem of constructing explicit cosmologies based on strongly coupled Higgs branch dynamics for elsewhere.

\footnotetext{
${ }^{4}$ The $F^{*} F$ term now has constant coefficient and therefore does not effect the equations of motion.
} 


\section{Conclusions and Open Questions}

We have described properties of the supergravity background dual to parts of the mixed Coulomb-Higgs branch of eight supercharge Yang-Mills theories with fundamental representations. One outcome of this work is a new set of constraints on unknown terms in the non-Abelian Dirac-Born-Infeld action in a curved background, which follow from the one to one correspondence between instantons and the Higgs branch, together with the non-renormalization of the metric on the Higgs branch. These constraints can be used to check future calculations of higher dimension operators in the Dirac-Born-Infeld action or, perhaps, to actually determine these terms. The latter possibility will require additional information, such as supersymmetry, since the number of constraints arising from the Higgs branch-Instanton correspondence is fewer than the number of unknown terms.

Without knowing the higher dimension operators in the Dirac-Born-Infeld action, one can still study the low energy dynamics of the Higgs branch at leading order in a large 't Hooft coupling expansion. Using the supergravity description of the Higgs branch, we have shown that the geodesic, or "moduli space," approximation is not suitable to describe the dynamics of a Higgs expectation value rolling towards a singularity in the moduli space. In violation of predictions of the geodesic approximation, this process is sensitive to the Coulomb-branch moduli and exhibits deceleration.

We have demonstrated qualitative properties of motion on the Higgs branch by studying time dependent solutions of the Dirac-Born-Infeld equations of motion with instanton number one. For vanishing Coulomb-branch moduli, our analysis was imprecise, since the time dependent solutions we discussed were far from self dual. Such solutions are not classified by the ADHM construction and do not map in any obvious way to points on the Higgs branch. A more precise analysis would require integrating out modes in the Dirac-Born-Infeld action which are transverse to the instanton moduli space, in order to obtain the higher derivative effective action on the Higgs branch. At leading order in the large $N$ expansion, this amounts to summing tree level graphs.

Although the theories we have considered are in a Higgs phase, computing the effective action on the Higgs branch might also be an enlightening warmup for computing higher derivative terms of the chiral Lagrangian in a yet to be constructed string dual of QCD. Like QCD in the chiral limit, the theories we consider have a non-trivial moduli space. At present, supergravity backgrounds of "QCD-like" theories which have a massless meson akin to the $\eta^{\prime}$ at $N \rightarrow \infty$ have been found [30]- [35], although a holographically dual description of a theory with a spontaneously broken non-Abelian chiral flavor symmetry remains elusive.

The deceleration mechanisms we have found are potentially interesting from a cosmological point of view since, as pointed out in the context of Coulomb-branch dynamics $[7,8]$, deceleration can give slow roll inflation even when a steep potential is introduced. It would be interesting to see what sort of cosmological models arise from rolling Higgs moduli at strong coupling and large $N$. The results described here are a preliminary step in investigating this question. For this purpose, it would also be useful to know the higher derivative effective action on the Higgs branch. It is important to bear in mind that the effective action on the Higgs branch is not reliable in situations in which there is produc- 
tion of W-bosons or their superpartners which become light near the origin of the Higgs branch. We have argued that this does in fact occur for certain fixed Coulomb-branch moduli as one rolls to the origin of the Higgs branch. Determining the details of the particle production would require further analysis of the collapse discussed in [29]. More generally, a better understanding of particle production in time dependent processes for which field strengths on the $\mathrm{Dp}+4$-brane are not self-dual is desirable.

In the above discussion, we have always taken the number of $\mathrm{Dp}+4$-branes and dissolved Dp-branes to be fixed in the $N \rightarrow \infty$ limit, so that their backreaction can be neglected. The number of dissolved D3-branes has also been kept fixed in this limit, for the same reason. It would be interesting to relax this constraint. The ADHM constraints should also classify the supergravity solutions associated with the Higgs branch of the $\mathrm{Dp}-\mathrm{Dp}+4$ system in which all branes have been replaced with geometry. At present the fully localized supergravity solutions associated with the D2-D6 [36,37] and D3-D7 [38] system have been studied, although not on the Higgs branch.

\section{Acknowledgements}

I am especially grateful to S. Kovacs and B. Kulik, for participation in early stages of this work. I have also profited from conversations with J. Erdmenger, K. Peeters, M. Schmaltz and M. Zamaklar.

\section{Appendix: a few useful relations for 't Hooft ten- sors}

The 't Hooft tensor is defined by

$$
\eta_{m n}^{a}=-\eta_{n m}^{a}, \eta_{i j}^{a}=\epsilon_{a i j}, \eta_{i 4}^{a}=\delta_{i a}
$$

where

$$
m, n=1 \cdots 4, \quad i, j=1 \cdots 3, \quad a=1 \cdots 3 .
$$

It satisfies

$$
\begin{aligned}
& \eta_{m n}^{a}=\frac{1}{2} \epsilon_{m n r s} \eta_{r s}^{a} \\
& \eta_{m l}^{b} \eta_{n m}^{c} \epsilon_{b c a}=-2 \eta_{l n}^{a} \\
& \eta_{m l}^{b} \eta_{m s}^{b}=3 \delta_{l s} \\
& \eta_{m l}^{e} \eta_{n t}^{e}=\delta_{m n} \delta_{l t}-\delta_{m t} \delta_{l n} \\
& \epsilon_{a b c} \epsilon_{d e c} \eta_{m l}^{b} \eta_{m s}^{d} \eta_{n t}^{e}=\eta_{n s}^{a} \delta_{l t}-\delta_{l n} \eta_{t s}^{a}-3 \delta_{l s} \eta_{n t}^{a} \\
& \epsilon_{p q m n} \eta_{n l}^{a}+\epsilon_{p q l n} \eta_{n m}^{a}=-2 \epsilon^{a b c} \eta_{p l}^{b} \eta_{q m}^{c}
\end{aligned}
$$




\section{References}

[1] J. M. Maldacena, "The large N limit of superconformal field theories and supergravity," Adv. Theor. Math. Phys. 2, 231 (1998) [Int. J. Theor. Phys. 38, 1113 (1999)] arXiv:hep-th/9711200.

[2] S. S. Gubser, I. R. Klebanov and A. M. Polyakov, "Gauge theory correlators from non-critical string theory," Phys. Lett. B 428, 105 (1998) arXiv:hep-th/9802109.

[3] E. Witten, "Anti-de Sitter space and holography," Adv. Theor. Math. Phys. 2, 253 (1998) arXiv:hep-th/9802150.

[4] Z. Guralnik, S. Kovacs and B. Kulik, "Holography and the Higgs branch of N = 2 SYM theories," arXiv:hep-th/0405127.

[5] P. C. Argyres, M. R. Plesser and N. Seiberg, "The Moduli Space of N=2 SUSY QCD and Duality in N=1 SUSY QCD," Nucl. Phys. B 471, 159 (1996) arXiv:hep-th/9603042.

[6] D. Kabat and G. Lifschytz, "Gauge theory origins of supergravity causal structure," JHEP 9905, 005 (1999) arXiv:hep-th/9902073.

[7] E. Silverstein and D. Tong, "Scalar speed limits and cosmology: Acceleration from D-cceleration," arXiv:hep-th/0310221.

[8] M. Alishahiha, E. Silverstein and D. Tong, "DBI in the sky," arXiv:hep-th/0404084.

[9] X. g. Chen, "Multi-throat brane inflation," Phys. Rev. D 71, 063506 (2005) arXiv:hep-th/0408084.

[10] A. Fayyazuddin and M. Spalinski, "Large N superconformal gauge theories and supergravity orientifolds," Nucl. Phys. B 535, 219 (1998) arXiv:hep-th/9805096.

[11] O. Aharony, A. Fayyazuddin and J. M. Maldacena, "The large N limit of N $=2,1$ field theories from three-branes in F-theory," JHEP 9807, 013 (1998) arXiv:hep-th/9806159.

[12] M. F. Atiyah, N. J. Hitchin, V. G. Drinfeld and Y. I. Manin, "Construction Of Instantons," Phys. Lett. A 65, 185 (1978).

[13] M. R. Douglas, "Gauge Fields and D-branes," J. Geom. Phys. 28, 255 (1998) arXiv:hep-th/9604198.

[14] E. Witten, "Small Instantons in String Theory," Nucl. Phys. B 460, 541 (1996) arXiv:hep-th/9511030.

[15] N. Dorey, T. J. Hollowood, V. V. Khoze and M. P. Mattis, "The calculus of many instantons," Phys. Rept. 371, 231 (2002) arXiv:hep-th/0206063.

[16] A. Karch and E. Katz, "Adding flavor to AdS/CFT," JHEP 0206, 043 (2002) arXiv:hep-th/0205236. 
[17] M. R. Douglas, "Branes within branes," arXiv:hep-th/9512077.

[18] N. Itzhaki, J. M. Maldacena, J. Sonnenschein and S. Yankielowicz, "Supergravity and the large N limit of theories with sixteen supercharges," Phys. Rev. D 58, 046004 (1998) arXiv:hep-th/9802042.

[19] K. Skenderis and M. Taylor, "Branes in AdS and pp-wave spacetimes," JHEP 0206, 025 (2002) arXiv:hep-th/0204054.

[20] M. B. Green, J. A. Harvey and G. W. Moore, "I-brane inflow and anomalous couplings on D-branes," Class. Quant. Grav. 14, 47 (1997) arXiv:hep-th/9605033.

[21] M. Wijnholt, "On curvature-squared corrections for D-brane actions," arXiv:hep-th/0301029.

[22] A. R. Frey, "String theoretic bounds on Lorentz-violating warped compactification," JHEP 0304, 012 (2003) arXiv:hep-th/0301189.

[23] P. Koerber and A. Sevrin, "The non-abelian D-brane effective action through order alpha'**4," JHEP 0210, 046 (2002) arXiv:hep-th/0208044.

[24] D. J. Gross and E. Witten, "Superstring Modifications Of Einstein's Equations," Nucl. Phys. B 277, 1 (1986).

[25] A. A. Tseytlin, "Vector Field Effective Action In The Open Superstring Theory," Nucl. Phys. B 276, 391 (1986) [Erratum-ibid. B 291, 876 (1987)].

[26] A. A. Tseytlin, "On non-abelian generalisation of the Born-Infeld action in string theory," Nucl. Phys. B 501, 41 (1997) arXiv:hep-th/9701125.

[27] E. A. Bergshoeff, A. Bilal, M. de Roo and A. Sevrin, "Supersymmetric non-abelian Born-Infeld revisited," JHEP 0107, 029 (2001) arXiv:hep-th/0105274.

[28] N. S. Manton, "A Remark On The Scattering Of Bps Monopoles," Phys. Lett. B 110, 54 (1982).

[29] P. Bizon, Y. N. Ovchinnikov and I. M. Sigal, "Collapse of an instanton," arXiv:math-ph/0307026.

[30] J. Babington, J. Erdmenger, N. J. Evans, Z. Guralnik and I. Kirsch, "Chiral symmetry breaking and pions in non-supersymmetric gauge / gravity duals," Phys. Rev. D 69, 066007 (2004) arXiv:hep-th/0306018.

[31] J. Babington, J. Erdmenger, N. J. Evans, Z. Guralnik and I. Kirsch, "A gravity dual of chiral symmetry breaking," Fortsch. Phys. 52, 578 (2004) arXiv:hep-th/0312263.

[32] M. Kruczenski, D. Mateos, R. C. Myers and D. J. Winters, "Towards a holographic dual of large-N(c) QCD," JHEP 0405, 041 (2004) arXiv:hep-th/0311270.

[33] J. L. F. Barbon, C. Hoyos, D. Mateos and R. C. Myers, "The holographic life of the eta'," JHEP 0410, 029 (2004) arXiv:hep-th/0404260. 
[34] N. J. Evans and J. P. Shock, "Chiral dynamics from AdS space," Phys. Rev. D 70, 046002 (2004) arXiv:hep-th/0403279.

[35] K. Ghoroku and M. Yahiro, "Chiral symmetry breaking driven by dilaton," Phys. Lett. B 604, 235 (2004) arXiv:hep-th/0408040.

[36] S. A. Cherkis and A. Hashimoto, "Supergravity solution of intersecting branes and AdS/CFT with flavor," JHEP 0211, 036 (2002) arXiv:hep-th/0210105.

[37] J. Erdmenger and I. Kirsch, "Mesons in gauge / gravity dual with large number of fundamental fields," arXiv:hep-th/0408113.

[38] B. A. Burrington, J. T. Liu, L. A. Pando Zayas and D. Vaman, "Holographic duals of flavored $\mathrm{N}=1$ super Yang-Mills: Beyond the probe approximation," arXiv:hep-th/0406207. 